\title{
Estimation of diabetes risk in Brazilian population by typing for polymorphisms in HLA-DR-DQ, INS and CTLA-4 genes
}

\author{
Omar M. Hauache ${ }^{\mathrm{a}, \mathrm{b}}$, André F. Reis ${ }^{\mathrm{a}, \mathrm{b}}$, Carolina S.V. Oliveira ${ }^{\mathrm{a}}$, José Gilberto H. Vieira ${ }^{\mathrm{a}, \mathrm{b}}$, \\ Minna Sjöroos ${ }^{\mathrm{c}}$ and Jorma Ilonen ${ }^{\mathrm{d}, *}$ \\ ${ }^{a}$ Escola Paulista de Medicina/UNIFESP, Sao Paulo, SP, Brazil \\ ${ }^{\mathrm{b}}$ Fleury Institute, Sao Paulo, SP, Brazil \\ ${ }^{\mathrm{c}}$ PerkinElmer Life and Analytical Sciences, Turku, Finland \\ d JDRF Centre for Prevention of Type 1 Diabetes in Finland and Department of Virology, University of Turku, \\ Turku, Finland
}

\begin{abstract}
The study aimed to further characterise HLA encoded risk factors of type 1 diabetes (T1D) in Brazilian population and test the capability of a low resolution full-house DR-DQ typing method to find subjects at diabetes risk. Insulin and CTLA-4 gene polymorphisms were also analysed. The method is based on an initial DQB1 typing supplemented by DQA1 and DR4 subtyping when informative. Increased frequencies of both (DR3)-DQA1*05-DQB $1 * 02$ and DRB1*04-DQA1*03-DQB1*0302 haplotypes were detected among patients. DRB $1 * 0401, * 0402, * 0404$ and $* 0405$ alleles were all common in DQB $1 * 0302$ haplotypes and associated with T1D. (DRB1*11/12/1303)-DQA1*05-DQB1*0301, (DRB1*01/10)-DQB1*0501, (DRB1*15)-DQB1*0602 and (DRB1*1301)-*0603 haplotypes were significantly decreased among patients. Genotypes with two risk haplotypes or a combination of a susceptibility associated and a neutral haplotype were found in 78 of 126 (61.9\%) T1D patients compared to 8 of $75(10.7 \%)$ control subjects $(P<0.0001)$. Insulin gene $-2221 \mathrm{C} / \mathrm{T}$ polymorphism was also associated with diabetes risk: CC genotype was found among $83.1 \%$ of patients compared to $69.3 \%$ of healthy controls $(P=0.0369$, OR 1.98) but CTLA-4 gene $+49 \mathrm{~A} / \mathrm{G}$ polymorphism did not significantly differ between patients and controls. Despite the diversity of the Brazilian population the screening sensitivity and specificity of the used method for T1D risk was similar to that obtained in Europe.
\end{abstract}

\section{Introduction}

Type 1 diabetes is a result of immune mediated destruction of insulin producing beta-cells in pancreatic islets. The central role of HLA region or human MHC associated genetic susceptibility has been known for thirty years and polymorphisms of class II region encoded molecules, especially DQ molecules, seem to be the single most important genetic disease risk determinant. In addition several loci in other chromosomes are contributing to the genetic risk, but only the ef-

* Corresponding author: Jorma Ilonen, University of Turku/ Medicity, Tykistökatu 6A, Turku, Finland. Tel.: +358 23337028 ; Fax: +358 2 3337000; E-mail: jorma.ilonen@utu.fi. fects of insulin and perhaps CTLA-4 gene region polymorphisms can be considered as firmly established [1]. DQ molecules associated with different strength with both disease risk and protection against it can be discerned, and interaction of the molecules encoded by the genotype is important as demonstrated by a clear dominant effect of strong protective molecules. In addition to HLA-DQ molecules there are other loci within HLA region affecting disease susceptibility. The role of DR molecules on DQ mediated susceptibility has been clearly demonstrated in DQA $1 * 03-\mathrm{DQB} 1 * 0302$ positive DR4 haplotypes where some particular DR4 subtype encoding alleles are associated with disease risk and others are abolishing the DQA $1 * 03-\mathrm{DQB} 1 * 0302$ associated susceptibility. There is also evidence for the 
role of class I alleles or loci in close vicinity in certain haplotypes as well as multiple other loci like DP, TNF region and a locus telomeric to HLA close to D6S2223 microsatellite marker [2-6].

MHC is the most polymorphic mammalian gene region and gene density there is extremely high. Complete high-resolution typing for HLA region is practically impossible, although much effort is made in tissue transplantation for exact matching of the donor recipient pairs for all molecules important in possible induction of immunological rejection.

Estimation of HLA related genetic risk for type 1 diabetes is at the moment used for selection of subjects for clinical prevention studies and studies aiming to identify environmental factors contributing to the betacell damaging autoimmune process $[7,8]$, but in future the identification of individuals at risk might be part of clinical routine if preventive measures will be developed. Study subjects in these projects have been either selected among first degree relatives or among general population. In the latter case the need for a simple and inexpensive screening protocol is accentuated as great number of samples must be processed. In addition to the huge polymorphism of HLA gene region it is also characterised by strong linkage disequilibrium, which makes testing for disease risk associated determinants more simple. Recombinations between DRB1, DQA1 and DQB1 genes are extremely rare and alleles in these loci are found in fixed combinations. Variability in major allele combinations still exists between various populations due to some ancient recombinatory events. Definition of either DRB1 or DQB1 alleles is in several haplotypes thus enough for low resolution typing, whereas in other cases more diverse combinations exist. For purposes of diabetes screening an emphasis can be put on polymorphisms known to be relevant for disease susceptibility.

We have developed a semiautomated sequence specific oligonucleotide hybridisation method for diabetes screening based on microtitre plate format and timeresolved fluorometry reading of hybridisation reactions of lanthanide chelate labelled oligonucleotides. PCR amplification of gene regions of interest directly from punched pieces of dried blood spots makes DNA extraction unnecessary and use of various lanthanide labels allows simultaneous measurement of three different signals in each microtitre plate well. The method was originally developed for testing the presence of T1D associated DQB $1 * 02$ and DQB $1 * 0302$ as well as protective DQB $1 * 0301$ and DQB $1 * 0602 / 3$ alleles [9] and was used in the Finnish Diabetes Prediction and
Prevention (DIPP) study [7]. It has later on been applied also for typing DQA1 alleles informative for diabetes risk when used in combination with DQB1 data and also for relevant DR4 subtypes [10]. These additional steps have been shown to increase the sensitivity and specificity of risk estimation considerably especially in more diverse Greek and Hungarian populations, whereas the effect in the less diverse Finnish populations was only modest [11]. Addition of a series of new DQB1 probes made possible a low-resolution full-house genotyping of all common Caucasian haplotypes $[12,13]$. An assay based on similar technology has also been developed for typing of insulin gene and CTLA-4 polymorphisms [14]. In the present study we aimed to investigate the capability of the methodology in estimation of T1D risk in one of the most diverse human populations, that in Brazil.

\section{Subjects and methods}

There were 126 patients diagnosed with type 1 diabetes, 72 males and 54 females. They were recruited in the out-patient Diabetes Center of UNIFESP-S?o Paulo, characterized by the presence of early-onset ketosis prone diabetes and insulin dependence directly after diagnosis. As controls, we studied a group of 75 unrelated non diabetic subjects without family history of diabetes recruited from the same area. Mean age at onset in patients was 11.7 (SD 4.7) years. 86 of the patients were white, 17 black, 19 mulatto, 3 oriental and one of unknown racial group. As controls, we studied a group of 75 unrelated non diabetic subjects without family history of diabetes recruited from the same area and with a similar racial distribution.

HLA genotyping was made by panels of sequence specific oligonucleotide probes hybridised with amplified polymorphic regions of DQB1, DQA1 and DRB1 genes. One of the primers was biotinylated and amplicons were transferred and fixed on streptavidin coated microtitre plate wells where they were denatured and hybridised with oligonucleotides. Twelve DQB1 probes, 3 DQA1 probes and 6 DRB1 probes were used in mixes of three probes labelled with various lanthanide (Europium, Samarium and Terbium) chelates. Specific signals for each label were measured after washes and addition of enhancement solution by timeresolved fluorometry. Details of the method including primer and probe sequences have been described earlier $[9,10,12]$. Similar method was used for genotyp- 
ing of insulin gene $-2221 \mathrm{C} / \mathrm{T}$ and CTLA-4 $+49 \mathrm{~A} / \mathrm{G}$ polymorphisms [14].

Differences in haplotype and genotype frequencies between patients and controls were compared using Chi-square test with Yates' correction and odds ratios (OR) calculated according to the formula: $\mathrm{a} / \mathrm{b} \times \mathrm{d} / \mathrm{c}$ in which a and $c$ are the numbers of marker positive patients and control subjects, and $\mathrm{b}$ and $\mathrm{d}$ are the numbers of marker negative patients and control subjects. Positive predictive value (PPV) was calculated according to formula:

sensitivity $\times$ prevalence / sensitivity $\times$ prevalence $+(1-$ specificity $) \times(1-$ prevalence $)$

and negative predicive value according to formula:

specificity $\times(1-$ prevalence $) /(1-$ sensitivity $)$

$\times$ prevalence + specificty $\times(1-$ prevalence $)$

\section{Results}

All samples were analysed for the presence of DQB1, DQA1 and DR4 alleles detectable with the used oligonucleotide panels. Haplotypes of alleles in different loci were deduced based on the known existing allelic associations. Table 1 lists separately haplotypes detected at least twice among either T1D patients or control subjects. DQA1 and DR4 subtyping reagents were designed for the specific purpose of detecting alleles known to characterise haplotypes differentially associated with diabetes risk and thus the level of resolution was variable between haplotypes. Allele definitions based on deduction are expressed within parentheses in the following text.

HLA-(DRB $1 * 03)-D Q A 1 * 05-D Q B 1 * 02$ and DRB $1 *$ 04-DQA $1 * 03-\mathrm{DQB} 1 * 0302$ haplotypes were highly significantly increased among T1D patients. HLA(DRB1*03)-DQA1*05-DQB1*02 haplotype was found in $60 \%$ of patients reflecting its high frequency also in background population. The frequency of DRB $1 * 04-\mathrm{DQB} 1 * 0302$ haplotypes was $12 \%$ among controls, but nearly half of patients still were positive. Odds ratio for DRB $1 * 04-\mathrm{DQB} 1 * 0302$ haplotypes was slightly higher than that of (DRB1*03)-DQA1*05DQB1*02. All major DRB1*04 subtypes usually associated with diabetes susceptibility, DRB $1 * 0401$, $* 0402, * 0404$ and $* 0405$ were increased and common among T1D patients, DRB1*0403/6 known to be protective was not found among patients, but was also present in only two healthy controls and the difference was thus not significant.
(DRB1*01/10)-DQB $1 * 0501, \quad(\mathrm{DRB} 1 * 11 / * 12 / * 130$ 3)-DQA $1 * 05-\mathrm{DQB} 1 * 0301$ and (DRB $1 * 15)-\mathrm{DQB} 1 * 06$ 02 were the most common haplotypes found in background population and they were all significantly decreased among T1D cases. (DRB1*1301)DQB $1 * 0603$ was also significantly decreased.

When the genotypes among T1D patients and controls were compared, the number of samples with each individual genotype became small due to the limited number of studied samples. (DRB1*03)-DQA1*05DQB1*02/DRB1*0401/2/4/5-DQA1*03-DQB1*0302 genotype was, however, associated with a very strong disease risk, $26.2 \%$ of patients were positive compared to none of the studied controls with this genotype (OR 54.1, $P<0.0001$ ). Homozygosity for HLA(DRB1*03)-DQA1*05-DQB1*02 haplotype was also significantly increased, $9.5 \%$ among patients compared to $1.3 \%$ of controls $(P=0.0468$, OR 5.42). (DRB1*03)-DQA1*05DQB1*02/ (DR11/12/1303)-D QA1*05-DQB1*0301 genotype was the only individual genotype, which was significantly decreased among patients (0\% vs. $6.7 \%, P=0.0135)$. When genotypes formed by five most common haplotypes were analysed for Hardy-Weinberg equilibrium among T1D cases the distribution differed significantly from the expected (Chi square 34.037, $d f=14, P<$ 0.005). Combination of two different HLA risk haplotypes, (DRB1*03)-DQA1*05-DQB1*02/DRB1*04DQA1*03-DQB1*0302, was more common than expected (Obs. 34, Exp. 22.3, Chi-square 6.074) and homozygosity for DRB1*04-DQB1*0302 less common than expected (Obs. 2, Exp. 8.1, Chi-square 4.619). Genotypes produced by the five most common haplotypes within control group were instead in HardyWeinberg equilibrium (Chi square 17.563, df $=14$, n.s.).

For further analysis of genotype effect we classified the haplotypes to susceptibility associated, neutral and protective. This classification was based on earlier literature $[13,15-18]$ and on the findings of this study. HLA-(DRB1*03)-DQA1*05-DQB1*02 and DRB $1 * 0401 / 2 / 4 / 5-\mathrm{DQA} 1 * 03-\mathrm{DQB} 1 * 0302$ haplotypes together with DRB1*0405-DQA1*03-DQB1*02 were thus classified as susceptibility haplotypes (S), all haplotypes found significantly decreased in this study (Table 1) as well as (DRB1*07)-DQA1*0201DQB1*0303, (DRB1*14)-DQB1*0503, and DRB1*04 03-DQA1*03-DQB1*0302 were classified as protective. These three last mentioned haplotypes were rare among the studied population and significant differences were not seen. The results of this risk classifica- 
Table 1

Frequency of subjects positive for defined HLA-DR-DQ haplotypes among diabetic patients and healthy controls

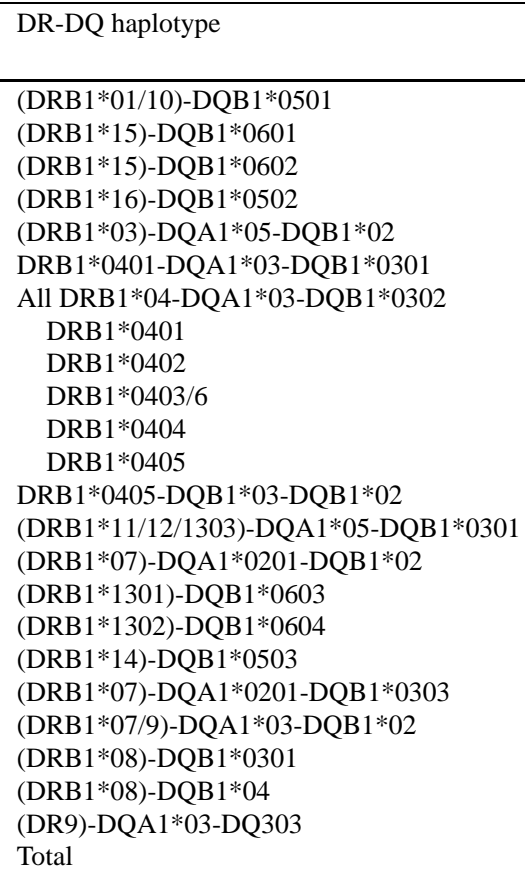

\begin{tabular}{rrrrcc}
\multicolumn{2}{c}{ T1D } & \multicolumn{2}{c}{ Controls } & OR & P \\
$\mathrm{N}$ & $\%$ & $\mathrm{~N}$ & $\%$ & & \\
20 & 15.9 & 26 & 34.7 & 0.36 & 0.0038 \\
0 & 0.0 & 3 & 4.0 & & \\
6 & 4.8 & 15 & 20.0 & 0.20 & 0.0015 \\
9 & 7.1 & 3 & 4.0 & & \\
76 & 60.3 & 15 & 20.0 & 6.08 & $<0.0001$ \\
1 & 0.8 & 1 & 1.3 & & \\
62 & 49.2 & 9 & 12.0 & 7.10 & $<0.0001$ \\
14 & 11.1 & 1 & 1.3 & 9.25 & 0.0229 \\
18 & 14.3 & 3 & 4.0 & 4.00 & 0.0386 \\
0 & 0.0 & 2 & 2.7 & & \\
8 & 6.3 & 0 & 0.0 & & \\
21 & 16.7 & 3 & 4.0 & 4.80 & 0.0141 \\
2 & 1.6 & 0 & 0.0 & & \\
12 & 9.5 & 20 & 26.7 & 0.29 & 0.0026 \\
13 & 10.3 & 12 & 16.0 & & \\
3 & 2.4 & 8 & 10.7 & 0.20 & 0.0293 \\
8 & 6.3 & 3 & 4.0 & & \\
3 & 2.4 & 3 & 4.0 & & \\
1 & 0.8 & 4 & 5.3 & & \\
5 & 4.0 & 2 & 2.7 & & \\
3 & 2.4 & 3 & 4.0 & & \\
6 & 4.8 & 6 & 8.0 & & \\
1 & 0.8 & 2 & 2.7 & & \\
126 & & 75 & & &
\end{tabular}

tion are shown in Table 2. Distribution of genotypes is clearly different between groups and one can see a risk gradient dependent on the haplotypic combination. A very high risk is associated with the presence of two risk haplotypes.Positive predictive value (PPV) for this genotype is $3.4 \%$ based on prevalence of $0.12 \%$ of T1D in Brazilian population of 15 years children. This is calculated from the yearly incidence of 8.0/100,000 children reported from Sao Paulo [19]. The presence of only one risk haplotype is associated with much lesser risk and this is further reduced by the simultaneous presence of a protective haplotype. $61.9 \%$ of T1D patients were found to belong to the two highest risk genotypes compared to only $10.7 \%$ of background population and PPV for this genotype group would be $0.69 \%$ and negative predictive value (NPV) $99.95 \%$. At least one of the haplotypes associated with disease susceptibility was found among $84.9 \%$ of diabetes patients compared to $29.3 \%$ of controls (PPV 0.34\%, NPV 99.97\%).

Polymorphisms of insulin gene region and CTLA-4 gene were analysed using $-2221 \mathrm{C} / \mathrm{T}$ and $+23 \mathrm{~A} / \mathrm{G}$ polymorphisms shown to be in association with disease risk in several populations. Tables 3 and 4 summarise the results. Insulin gene $-2221 \mathrm{CC}$ genotype was significantly increased among T1D patients, but no significant differences were found in CTLA-4 genotypes

(Table 4). No significant differences were seen in the frequency of these markers between patients divided to high and low risk genotypes ( $\mathrm{S} / \mathrm{S}$ and $\mathrm{S} / \mathrm{N}$ vs others, data not shown). Distribution of INS and CTLA-4 genotypes were in Hardy-Weinberg equilibrium in both groups.

\section{Discussion}

Brazilian population with its mixed ancestry is an especially diverse one and thus able to give some special insights to research of diseases with a genetic component. Variability of allelic associations makes genetic disease risk studies also demanding as markers used because of their linkage to disease risk genes themselves may not necessarily be useful to the extent found in more homogeneous populations.

In the present study we could detect as expected the strong association of the two major diabetes risk haplotypes, DRB $1 * 03-\mathrm{DQB} 1 * 05-\mathrm{DQB} 1 * 02$ and DRB1*04DQA1*03-DQB1*0302 with the disease. DR3 and DR4 associations were detected already in serological studies in Brazilian population [20] and confirmed later on by sequence based typing [21,22]. Similarly the protective effect of DR15(2) and respectively, 
Table 2

Frequency of genotypes consisting of susceptibility associated (S), neutral $(\mathrm{N})$ and protective $(\mathrm{P})$ haplotypes among patients with type 1 diabetes and control subjects. Positive predictive value (PPV) and negative predictive value (NPV) were calculated based on a prevalence of $0.12 \%$

\begin{tabular}{lrrrrrrr} 
Genotype & \multicolumn{2}{c}{ T1D } & \multicolumn{2}{c}{ Controls } & O.R. & PPV & NPV \\
& $\mathrm{N}$ & \multicolumn{1}{c}{$\%$} & $\mathrm{~N}$ & $\%$ & & & \\
S/S & 49 & 38.9 & 1 & 1.3 & 47.09 & 3.39 & 99.93 \\
S/N & 29 & 23.0 & 7 & 9.3 & 2.90 & 0.30 & 99.90 \\
S/P & 27 & 21.4 & 14 & 18.7 & 1.19 & 0.14 & 99.88 \\
N/N & 6 & 4.8 & 7 & 9.3 & 0.49 & 0.06 & 99.87 \\
P/N & 9 & 7.1 & 21 & 28.0 & 0.20 & 0.03 & 99.85 \\
P/P & 6 & 4.8 & 25 & 33.3 & 0.10 & 0.02 & 99.83 \\
Total & 126 & & 75 & & & &
\end{tabular}

$P<0.0001, d f=5$.

DRB1*1501-DQB1*0602, was detected in all these earlier studies.

The study based on 56 families with a diabetic child by Volpini and colleagues confirmed the risk conferred by DRB $1 * 03-\mathrm{DQA} 1 * 05-\mathrm{DQB} 1 * 02$ and DRB $1 * 04-D Q A 1 * 03-D Q B 1 * 0302$ haplotypes, but the role of DRB $1 * 0401$ subtype among the transmitted DR4 haplotypes was prominent [21]. We could instead detect the association of all other common DR4 subtypes than DRB1*0403/6 with disease susceptibility. The increase of DRB1*0404 allele was only of borderline significance, but it was not at all detected among healthy controls. The numbers do not really allow a more thorough estimation of relative strength of disease risk between various DRB $1 * 04$ alleles, but suggest strong association with each one except DRB $1 * 0403 / 06$. This allele was rare also among control population although its lack among diabetic cases is in line with protective effect confirmed in numerous studies [23]. Findings reported by Fernandes and coworkers support our results although findings for no DR4 subtypes were significant due to the small numbers in the patient series of 64 cases [22].

Volpini et al. suggested that the lack of the protective effect of DRB1*15-DQA1*0102-DQB1*0602 would be a peculiar characteristic of Brazilian population [21]. This was probably a chance effect in a small sample series as we found this haplotype in fact to have the strongest protective effect. Similarly did Fernandes and coworkers find a significant decrease of DRB $1 * 15$ and DQB1*0602/03 alleles [22].

The protection conferred by DRB1*13-DQB $1 * 0301$ and DRB 1*11-DQB 1*0301 found by Volpini et al. [21] was confirmed in our series although both haplotypes were detected as a group together with all DQA $1 * 05-$ DQB $1 * 0301$ positive haplotypes. DRB $1 * 01-\mathrm{DQB} 1 *$ 0501 haplotype was also protective in both studies.
Table 3

Genotypes of Insulin gene $-2221 \mathrm{C} / \mathrm{T}$ polymorphism among T1D patients and controls

\begin{tabular}{lrrrrrr} 
Genotype & \multicolumn{2}{c}{ T1D } & \multicolumn{2}{c}{ Controls } & OR & P \\
& $\mathrm{N}$ & $\%$ & $\mathrm{~N}$ & $\%$ & & \\
CC & 103 & 83.1 & 52 & 69.3 & 1.98 & 0.0369 \\
CT & 19 & 15.3 & 19 & 25.3 & & \\
TT & 2 & 1.6 & 4 & 5.3 & & \\
Total & 124 & & 75 & & &
\end{tabular}

Table 4

Genotypes of CTLA- 4 gene +49 A/G polymorphism among T1D patients and controls

\begin{tabular}{lcccc} 
Genotype & \multicolumn{2}{c}{ T1D } & \multicolumn{2}{c}{ Controls } \\
& $\mathrm{N}$ & $\%$ & $\mathrm{~N}$ & $\%$ \\
AA & 42 & 33.9 & 30 & 40.0 \\
AG & 63 & 50.8 & 34 & 45.3 \\
GG & 19 & 15.3 & 11 & 14.7 \\
Total & 124 & & 75 &
\end{tabular}

This haplotype was also the most common in background population and thus most affected by secondary decrease due to the increase of some haplotypes. In the present study we could also detect a significantly decreased frequency of (DRB1*1301)-DQB $1 * 0603$ haplotype. (DRB1*07)-DQA $1 * 0201-\mathrm{DQB} 1 * 02$ and (DR8)-DQB1*04 haplotypes were neutral like also DQA $1 * 03-D Q B 1 * 02$ haplotype apparently associated with DRB1*07 and/or DRB1*09 alleles as found in black Brazilian population [24]. Samples with this haplotype without DRB1*0405 were always found in black or mulatto patients.

(DRB1*07)-DQA $1 * 0201-\mathrm{DQB} 1 * 0303$ and (DRB1 *14)-DQB $1 * 0503$ haplotypes have been found strongly protective in several populations [13,16,25-28], but they were not found significantly decreased among T1D patients in this study. However, these were relatively rare also in the background population and a ten- 
dency to decreased frequency was found especially for (DRB1*07)-DQA1*0201-DQB1*0303 haplotype.

Classification of haplotypes to risk and protection associated or neutral ones allowed a simple risk grading system based on six possible genotypic combinations of haplotypes. Genotypes with two diabetes risk associated haplotypes are in fact all but one (DRB1*03)-DQA1*05-DQB1*02/DRB1*04DQB $1 * 03-\mathrm{DQB} 1 * 0302$ heterozygous demonstrating again the well known high risk associated with this particular genotype. More than $60 \%$ of patients can be found in the two highest risk genotype combinations, which were found in only $10.7 \%$ or controls. This demonstrates that the used HLA typing procedure is able to high extent predict T1D risk also in this diverse population. Sensitivity and specificity of HLA-DR-DQ based genotyping is in fact quite similar as described in several European populations analysed by somewhat varying genotyping schemes $[15,29,30]$. In spite of the fact that in our study groups there were few Africans and mulattos we think that it is representative of general Brazilian population. The result of five centuries of interethnic crosses of Europeans, Africans and autochthonous Amerindians have created an extremely heterogeneous population and studies of ancestry of mitochondrial DNA in mostly white Brazilians showed nearly equal amounts of Amerindian, African and European matrilineal genetic contribution [31].

Somewhat more refined disease risk grading might be possible also based on class II HLA typing, but larger patient and control series should be typed for that purpose. Genotyping for additional loci outside HLA might also offer possibilities to increase the efficiency of risk estimation. Insulin gene region and CTLA-4 gene are the two loci, which besides HLA can be listed among confirmed loci although their total contribution to diabetes risk, like also that of those remaining still to be mapped, is small compared to HLA. Analysis of INS gene $-2221 \mathrm{C} / \mathrm{T}$ polymorphism confirmed the disese association also in this population and the presence of homozygous risk genotype might be included into the risk estimation. OR associate with risk genotype is not high but could further define especially those groups with moderate HLA based diabetes risk. Another limiting factor for the usage of INS gene analysis is the high frequency of risk genotype among background population. The used marker of CTLA-4 polymorphism could not detect significant difference between patients and controls and its efficiency in risk estimation must thus be low.

\section{References}

[1] L.L. Field, Genetic linkage and association studies of Type I diabetes: challenges and rewards, Diabetologia 45 (2002), 21-35.

[2] P.H. Moghaddam, P. deKnijf, B.O. Roep, B. VanderAuwera, A. Naipal, F. Gorus, F. Schuit and M.J. Giphart, Genetic structure of IDDM1: Two separate regions in the major histocompatibility complex contribute to susceptibility or protection, Diabetes 47 (1998), 263-269.

[3] S. Nejentsev, Z. Gombos, A.P. Laine, R. Veijola, M. Knip, O. Simell, O. Vaarala, H.K. Akerblom and J. Ilonen, Nonclass IIHLA gene associated with type 1 diabetes maps to the 240-kb region near HLA-B, Diabetes 49 (2000), 2217-2221.

[4] P. Zavattari, R. Lampis, C. Motzo, M. Loddo, A. Mulargia, M. Whalen, M. Maioli, E. Angius, J.A. Todd and F. Cucca, Conditional linkage disequilibrium analysis of a complex disease superlocus, IDDM1 in the HLA region, reveals the presence of independent modifying gene effects influencing the type 1 diabetes risk encoded by the major HLA-DQB1,-DRB1 disease loci, Human Molecular Genetics 10 (2001), 881-889.

[5] B.A. Lie, J.A. Todd, F. Pociot, J. Nerup, H.E. Akselsen, G. Joner, K. Dahl Jorgensen, K.S. Ronningen, E. Thorsby and D.E. Undlien, The predisposition to type 1 diabetes linked to the human leukocyte antigen complex includes at least one non-class II gene, Am J Hum Genet 64 (1999), 793-800.

[6] H.A. Erlich, J.I. Rotter, J.D. Chang, S.J. Shaw, L.J. Raffel, W. Klitz, T.L. Bugawan and A. Zeidler, Association of HLADPB1(*)0301 with IDDM in Mexican-Americans, Diabetes 45 (1996), 610-614.

[7] A. Kupila, P. Muona, T. Simell, H. Arvilommi, H. Savolainen, A.-M. Hämäläinen, S. Korhonen, T. Kimpimäki, M. Sjöroos, J. Ilonen, M. Knip and O. Simell, Feasibility of genetic and immunological prediction of type 1 diabetes in a populationbased birth cohort, Diabetologia 44 (2001), 290-297.

[8] M. Rewers, T.L. Bugawan, J.M. Norris, A. Blair, B. Beaty, M. Hoffman, R.S. McDuffie, Jr., R.F. Hamman, G. Klingensmith, G.S. Eisenbarth and H.A. Erlich, Newborn screening for HLA markers associated with IDDM: diabetes autoimmunity study in the young (DAISY), Diabetologia 39 (1996), 807-812, Issn: 0012-186x.

[9] M. Sjöroos, A. Iitiä, J. Ilonen, H. Reijonen and T. Lövgren, Triple-label hybridization assay for type-1 diabetes-related HLA alleles, BioTechniques 18 (1995), 870-877.

[10] S. Nejentsev, M. Sjöroos, T. Soukka, M. Knip, O. Simell, T. Lövgren and J. Ilonen, Population based genetic screening for type 1 diabetes risk in Finland: Selective genotyping of the markers in the HLA-DQB1, -DQA1 and -DRB1 loci, Diabetic Med 16 (1999), 985-992.

[11] R. Hermann, C. Bartsocas, G. Soltesz, A. Vazeou, P. Paschou, E. Bozas, A. Malamitsi-Puchner, O. Simell, M. Knip and J. Iionen, Genetic screening for individuals at high risk for type 1 diabetes in the general population using class II alleles as disease markers. A comparison between three European populations with variable rates of disease incidence, Diab/Metabl Res Rev (2004), in press.

[12] M. Laaksonen, T. Pastinen, M. Sjöroos, S. Kuokkanen, J. Ruutiainen, M.-L. Sumelahti, H. Reijonen, R. Salonen, J. Wikström, M. Panelius, J. Partanen, P.J. Tienari and J. Ilonen, HLA class II associated risk and protection against multiple sclerosis - a Finnish family study, J. Neuroimmunol. 122 (2002), 140-145.

[13] R. Hermann, H. Turpeinen, A.P. Laine, R. Veijola, M. Knip, O. Simell, I. Sipila, H.K. Akerblom and J. Ilonen, HLA DR-DQ- 
encoded genetic determinants of childhood-onset type 1 diabetes in Finland: An analysis of 622 nuclear families, Tissue Antigens 62 (2003), 162-169.

[14] A.P. Laine, R. Hermann, M. Knip, O. Simell, H.K. Åkerblom and J. Ilonen, HLA genotype has a modest effect on the insulin gene polymorphism-associated susceptibility to Type 1 diabetes in the Finnish population, Tissue Antigens 63 (2004), 72-74.

[15] A.P. Lambert, K.M. Gillespie, G. Thomson, H.J. Cordell, J.A. Todd, E.A. Gale and P.J. Bingley, Absolute risk of childhoodonset type 1 diabetes defined by human leukocyte antigen class II genotype: a population-based study in the United Kingdom, J Clin Endocrinol Metab 89 (2004), 4037-4043.

[16] R.F. Schipper, B.P. Koeleman, G.J. Bruining, G.M. Schreuder, W. Verduijn, R.R. De Vries and B.O. Roep, HLA class II associations with Type 1 diabetes mellitus: a multivariate approach, Tissue Antigens 57 (2001), 144-150.

[17] F. Cucca, R. Lampis, F. Frau, D. Macis, E. Angius, P. Masile, M. Chessa, P. Frongia, M. Silvetti, A. Cao, S. Devirgiliis and M. Congia, The distribution of DR4 haplotypes in Sardinia suggests a primary association of type I diabetes with DRB1 and DQB1 loci, Hum. Immunol. 43 (1995), 301-308.

[18] B.O. Roep, R. Schipper, W. Verduyn, G.J. Bruining, G.M. Schreuder and R.R. de Vries, HLA-DRB1*0403 is associated with dominant protection against IDDM in the general Dutch population and subjects with high-risk DQA1*0301-DQB1*0302/DQA1*0501-DQB1*0201 genotype, Tissue Antigens 54 (1999), 88-90.

[19] M. Karvonen, M. Viik Kajander, E. Moltchanova, I. Libman, R. LaPorte and J. Tuomilehto, Incidence of childhood type 1 diabetes worldwide. Diabetes Mondiale (DiaMond) Project Group, Diabetes Care 23 (2000), 1516-1526.

[20] D.L. Eizirik, C.M. Monteiro, J.C. Voltarelli and M.C. Foss, Frequency of HLA antigens in a Brazilian type I diabetic population, Braz J Med Biol Res 20 (1987), 533-537.

[21] W.M.G. Volpini, G.V. Testa, S.B.D. Marques, L.I. Alves, M.E.R. Silva, S.A. Dib, G. Guerra, M. Paulino, S. Marini, L.B.L. Persoli and S. Caillat-Zucman, Family-based association of HLA class II alleles and haplotypes with type I diabetes in Brazilians reveals some characteristics of a highly diversified population, Human Immunology 62 (2001), 1226-1233.

[22] A.P. Fernandes, P. Louzada-Junior, M.C. Foss and E.A. Donadi, HLA-DRB1, DQB1, and DQA1 allele profile in Brazilian patients with type 1 diabetes mellitus, Ann N Y Acad Sci 958 (2002), 305-308.

[23] D.E. Undlien, T. Friede, H.G. Rammensee, G. Joner, K. Dahl-
Jorgensen, O. Sovik, H.E. Akselsen, I. Knutsen, K.S. Ronningen and E. Thorsby, HLA-encoded genetic predisposition in IDDM: DR4 subtypes may be associated with different degrees of protection, Diabetes 46 (1997), 143-149.

[24] M.E. Moraes, M. Fernandez-Vina, I. Salatiel, S. Tsai, J.R. Moraes and P. Stastny, HLA Class-II DNA Typing in two Brazilian Populations, Tissue Antigens 41 (1993), 238-242.

[25] A. Petrone, T.L. Bugawan, C.A. Mesturino, L. Nistico, A. Galgani, G. Giorgi, I. Cascino, H.A. Erlich, U. Di Mario and R. Buzzetti, The distribution of HLA class II susceptible/protective haplotypes could partially explain the low incidence of type 1 diabetes in continental Italy (Lazio region), Tissue Antigens 58 (2001), 385-394.

[26] J.A. Noble, A.M. Valdes, M. Cook, W. Klitz, G. Thomson and H.A. Erlich, The role of HLA class II genes in insulindependent diabetes mellitus: Molecular analysis of $180 \mathrm{Cau}-$ casian, multiplex families, American Journal of Human Genetics 59 (1996), 1134-1148.

[27] D.E. Undlien, I. Kockum, K.S. Ronningen, R. Lowe, C.B. Saanjeevi, J. Graham, B.A. Lie, H.E. Akselsen, A. Lernmark and E. Thorsby, HLA associations in type 1 diabetes among patients not carrying high-risk DR3-DQ2 or DR4-DQ8 haplotypes, Tissue Antigens 54 (1999), 543-551.

[28] O. Cinek, S. Kolouskova, M. Snajderova, Z. Sumnik, P. Sedlakova, P. Drevinek, J. Varinec and K.S. Ronningen, HLA class II genetic association of type 1 diabetes mellitus in Czech children, Pediatric Diabetes (2001), 98-102.

[29] R. Buzzetti, A. Galgani, A. Petrone, M.L. Del Buono, H.A. Erlich, T.L. Bugawan, R. Lorini, F. Meschi, G. Multari, P. Pozzilli, M. Locatelli, G. Bottazzo and U. Di Mario, Genetic prediction of type 1 diabetes in a population with low frequency of HLA risk genotypes and low incidence of the disease (the DIABFIN study), Diabetes Metab Res Rev 20 (2004), 137-143.

[30] R. Hermann, C.S. Bartsocas, G. Soltesz, A. Vazeou, P. Paschou, E. Bozas, A. Malamitsi-Puchner, O. Simell, M. Knip and J. Ilonen, Genetic screening for individuals at high risk for type 1 diabetes in the general population using HLA Class II alleles as disease markers. A comparison between three European populations with variable rates of disease incidence, Diabetes Metab Res Rev 20 (2004), 322-329.

[31] J. Alves-Silva, M. da Silva Santos, P.E. Guimaraes, A.C. Ferreira, H.J. Bandelt, S.D. Pena and V.F. Prado, The ancestry of Brazilian mtDNA lineages, Am J Hum Genet 67 (2000), 444-461. 


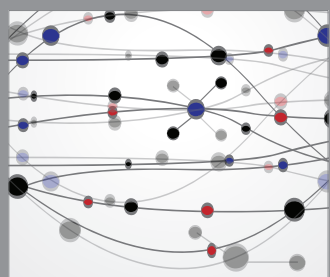

The Scientific World Journal
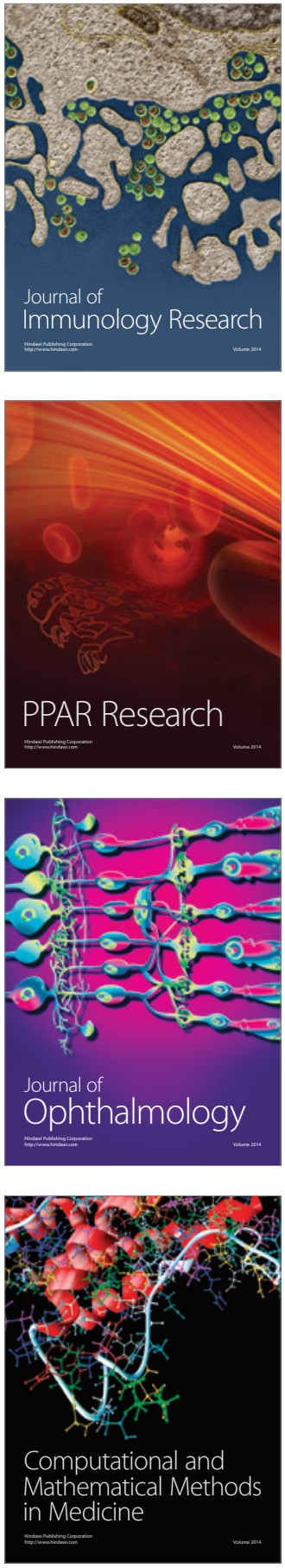

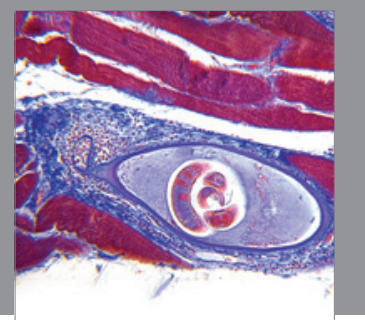

Gastroenterology

Research and Practice
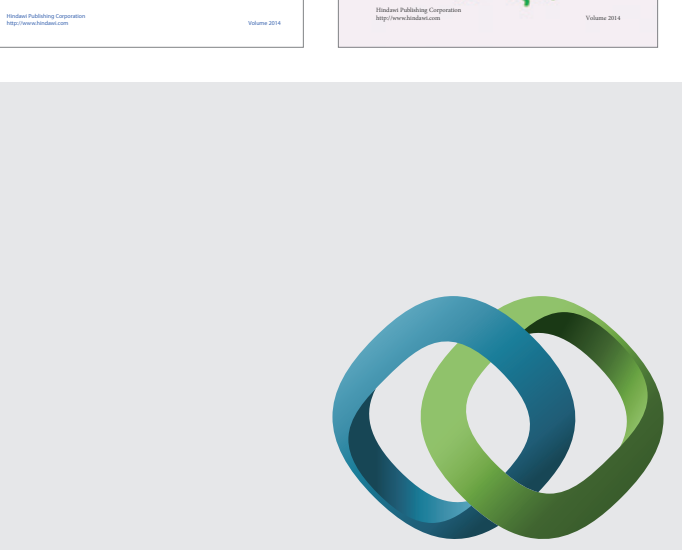

\section{Hindawi}

Submit your manuscripts at

http://www.hindawi.com
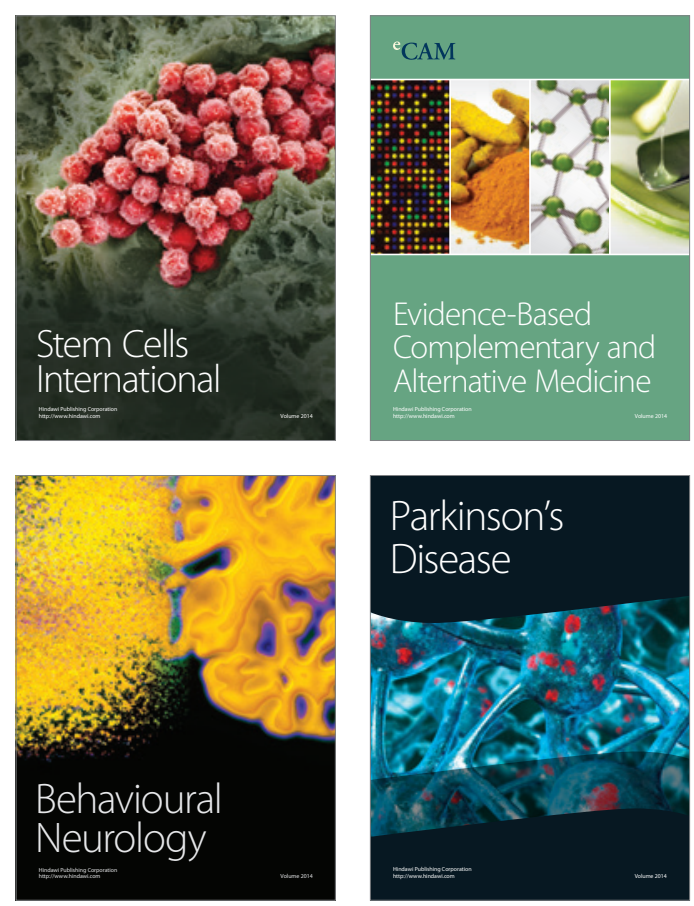

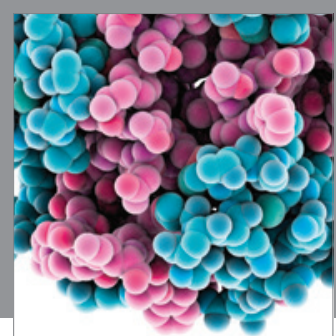

Journal of
Diabetes Research

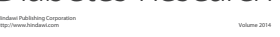

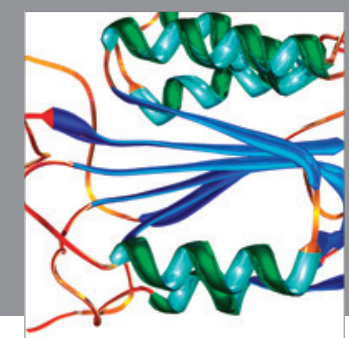

Disease Markers
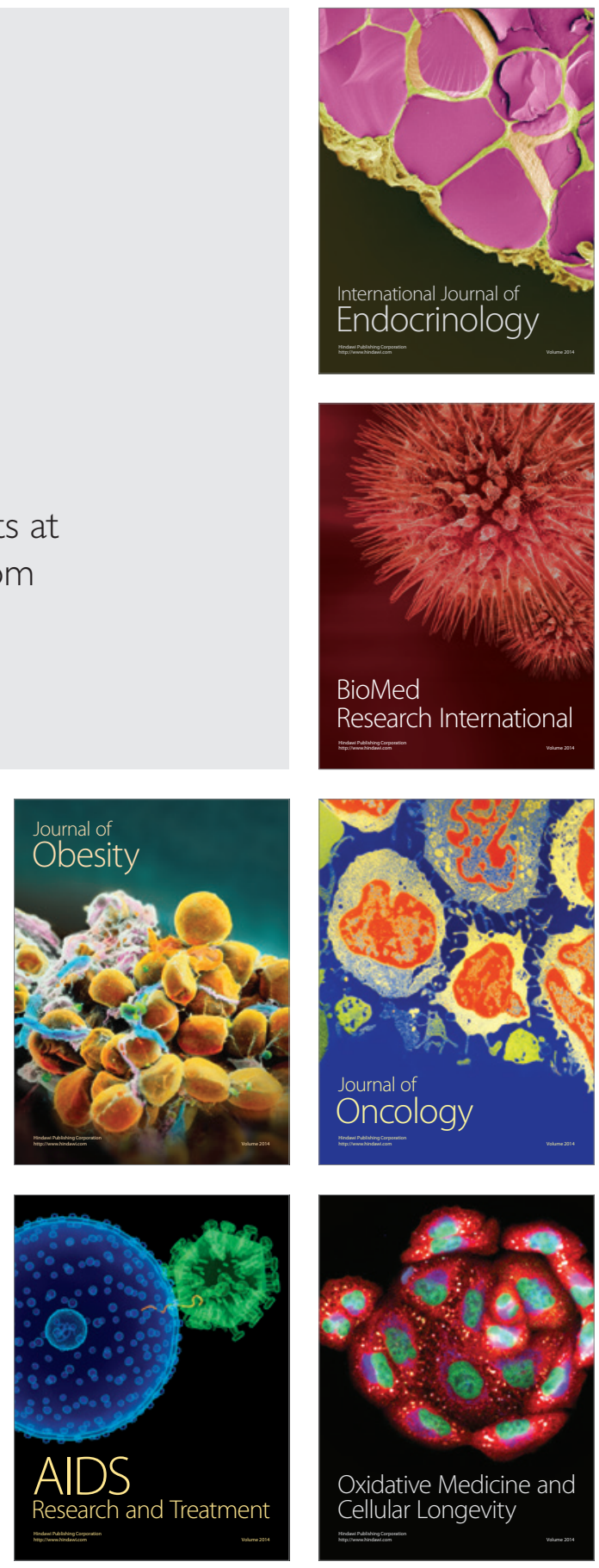